\title{
Two-dimensional Anisotropic Random Walks: Fixed versus Random Column Configurations for Transport Phenomena
}

\section{Endre Csáki}

A. Rényi Institute of Mathematics, Hung. Acad. Sci., Budapest, P.O.B. 127, H1364, Hungary. E-mail address: csaki.endre@renyi.mta.hu

\section{Miklós Csörgő}

School of Mathematics and Statistics, Carleton University, 1125 Colonel By Drive, Ottawa, Ontario, Canada K1S 5B6. E-mail address: mcsorgo@math.carleton.ca

\section{Antónia Földes}

Department of Mathematics, College of Staten Islands, CUNY, 2800 Victory Blvd., Staten Island, New York 10314, U.S.A. E-mail address: Antonia.Foldes@csi.cuny.edu

Pál Révész

Institut für Statistik und Wahrscheinlichkeitstheorie, Technische Universität Wien, Wiedner Hauptstrasse 8-10/107 A-1040 Vienna, Austria.

E-mail address: revesz.paul@renyi.mta.hu

\begin{abstract}
We consider random walks on the square lattice of the plane along the lines of Heyde (1982, 1993) and den Hollander (1994), whose studies have in part been inspired by the so-called transport phenomena of statistical physics. Twodimensional anisotropic random walks with anisotropic density conditions á la Heyde (1982, 1983) yield fixed column configurations and nearest-neighbour random walks in a random environment on the square lattice of the plane as in den Hollander (1994) result in random column configurations. In both cases we conclude simultaneous weak Donsker and strong Strassen type invariance principles in terms of appropriately constructed anisotropic Brownian motions on the plane, with selfcontained proofs in both cases. The style of presentation throughout will be that of a semi-expository survey of related results in a historical context.
\end{abstract}

Keywords Random walk; anisotropic lattices; random anisotropic lattices; anisotropic Brownian motions; in probability and almost sure stochastic approximations 


\section{Introduction}

Motivated by, and along the lines of, Heyde $(1982,1993)$ and den Hollander (1994), in this paper we consider random walks on the square lattice of the plane whose studies have in part been inspired by the so-called transport phenomena of statistical physics (cf., e.g., 1 Introduction in Heyde (1993) and 1.4 History of den Hollander (1994), and their references).

Two-dimensional anisotropic random walks with asymptotic density conditions á la Heyde $(1982,1983)$ yield fixed column configurations (cf. Section 1.1) and nearest-neighbour random walks in a random environment on the square lattice of the plane as in den Hollander (1994) result in random column configurations (cf. Section 1.2). In both cases, in the same respective sections, we conclude simultaneous weak Donsker and strong Strassen type invariance principles in terms of appropriately constructed anisotropic Brownian motions on the plane via making use of the initial approach of the above papers in hand.

Thus, in Section 1.1, via the initial approach of Heyde $(1992,1993)$, we arrive at the respective weak and strong fixed column embedding conclusions as in Theorem 1 and Theorem 2, holding simultaneously on the same probability space, while in Section 1.2 we conclude the respective corresponding simultaneous weak and strong random column conclusions of Theorem $\mathrm{C}$ and Theorem 3, via the initial approach of den Hollander (1994).

In view of this syncronized parallel relationship we may say that, to the extent of simultaneously having a weak Donsker and strong Strassen type asymptotic behaviour, under their respective initial conditions the two random walks in hand behave similarly. More precisely, in addition to extending the results of Heyde (1982, 1993) on the weak Donsker and strong Strassen type asymptotic path behaviour of a 2-dimensional anisotropic random walk with transition probabilities as in (1.1) under his asymptotic density conditions as in (1.2), the latter with $\eta=0$ in the case of Theorem 1, in this exposition we have also succeeded in showing that, to the extent of weak Donsker and strong Strassen type asymptotic path behaviour, a nearest-neighbour 2-dimensional random walk with probability law $\mu$ as in the $(\star)$ den Hollander (1994) condition of (1.17) and transition probabilities as in (1.19) behaves like a 2-dimensional anisotropic random walk with anisotropy determined only by $q$ as in (1.17) and not by any other parameters of $\mu$. This in turn also amounts to the main observation of this exposition (cf. Observation in Section 1.2).

The corresponding respective self contained proofs are given in Sections 2.1 and 2.2. The style of presentation throughout will be that of a semi-expository survey of related results in a historical context.

We also wish to note that, using an alternative construction of the random walk 
$\left\{X_{n}, Y_{n}\right\}, n=1,2, \ldots$, on $\mathbb{Z}^{2}$ with horizontal step transition probabilities $1 / 2-p_{j}$ and vertical step transition probabilities $p_{j}$, i.e., an inverted "upside down" version of transion probabilities as in (1) of Heyde (1993) (cf. (1.1) in this exposition), and assuming the asymptotic density condition of Heyde (1982) for the transition probabilities as spelled out in (1.2), Csáki et al. (2013) established a joint strong approximation result for $\left\{X_{n}, Y_{n}\right\}$ by two independent standard Wiener processes as in their Theorem 1.1. Mutatis mutandis, an alternative equivalent version of our Theorem 2 per se could also be proved via making use of the latter strong approximation result. On the other hand, our Donsker type in probability approximation as in Theorem 1 is new, and so is also its relationship to our present way of establishing Theorem 2 (cf. Remark 2).

Next we note that our strong Strassen type Theorem 3, an extended version of (2.1) of Theorem 2 in den Hollander (1994), is new and so is also its relationship under the same conditions to Therem C, a reformulated version of Theorem 1 of den Hollander (1994) á la our Theorem 1 (cf. Remark 5).

In the context of nearest-neighbour random walks in random environment on the square lattice on the plane, den Hollander (1994) also deals with other functions of the walk, like return probability and range. For anisotropic random walks on the plane Csáki et al. (2013) also study their local times, recurrence and range under various conditions. We have not succeeded in furthering and synchronizing the result yielded by the two approaches in hand along the latter lines.

\subsection{Two-dimensional anisotropic random walks; Fixed col- umn configurations with asymptotic density conditions}

For the sake of introducing and motivating the study of two-dimenssional anisotropic random walks on the square lattice $\mathbb{Z}^{2}$ we quote from 1. Introduction of Heyde (1993):

"One area of application involves conductivity of various organic salts, such as tetrathiofuvalene (TTF)-tetracyanoquinodimethane (TCNQ), which show signs of superconductivity. They conduct strongly in one direction but not others. Indeed, the conductivity parallel to the structural axis is 100 times or more that perpendicular to it. The molecular forms of TCNQ and TTF are planar so that they can be easily stacked and, in fact, the structure of the TTF-TCNQ crystal is generally thought to consist of parallel columns of separately stacked TTF and TCNQ molecules. Another related area of application concerns transport in physical systems which lack complete connectivity such as with fluid flow through packed columns, for example in gas absorption or distillation processes. 
In such situations gases or liquids predominantly flow through vertical cylinders filled with a random packing of inert objects such as ceramic rings.

The predominance of one dimension for the transport, say along rows, plus a possible lack of complete connectivity, can be modeled rather more generally by an anisotropic 2-dimensional random walk in which the transition mechanism depends only on the index of the column which is at present occupied. Thus, we consider a random walk which, if situated at a site on column $j$, moves with probability $p_{j}$ to either horizontal neighbour and with probability $\frac{1}{2}-p_{j}$ to either vertical neighbour at the next step."

More formally, let $X_{n}$ and $Y_{n}$ denote the horizontal and vertical positions of the walk after $n$ steps, starting from $X_{0}=Y_{0}=0$, with transition probabilities as in (1) of Heyde (1993):

$$
\begin{aligned}
& P\left\{\left(X_{n+1}, Y_{n+1}\right)=(j-1, k) \mid\left(X_{n}, Y_{n}\right)=(j, k)\right\}=p_{j} \\
& P\left\{\left(X_{n+1}, Y_{n+1}\right)=(j+1, k) \mid\left(X_{n}, Y_{n}\right)=(j, k)\right\}=p_{j} \\
& P\left\{\left(X_{n+1}, Y_{n+1}\right)=(j, k-1) \mid\left(X_{n}, Y_{n}\right)=(j, k)\right\}=1 / 2-p_{j} \\
& P\left\{\left(X_{n+1}, Y_{n+1}\right)=(j, k+1) \mid\left(X_{n}, Y_{n}\right)=(j, k)\right\}=1 / 2-p_{j}
\end{aligned}
$$

for $(j, k) \in \mathbb{Z}^{2}$, and $n=0,1,2, \ldots$, a so-called anisotropic 2-dimensional random walk with possibly unequal symmetric horizontal and vertical step transition probabilities that depend only on the index of the column which is at present occupied.

We assume throughout that $0<p_{j} \leq 1 / 2$ and $\min _{j \in \mathbb{Z}} p_{j}<1 / 2$.

Thus, as in Heyde (1993), we consider a random walk which, if situated at a site on column $j$, moves with probability $p_{j}$ to either horizontal neighbour, and with probability $1 / 2-p_{j}$ to either vertical neighbour at the next step.

The case $p_{j}=1 / 4, j=0, \pm 1, \pm 2, \ldots$ corresponds to a simple symmetric random walk on the plane. For studies of this case we refer to Erdös and Taylor (1960), Dvoretzky and Erdős (1951), Révész (2013) and the references in these works.

When $p_{j}=1 / 2$ for some $j$, then the vertical line $x=j$ is missing, not connected, i.e., we have a so-called non-connective column.

If all $p_{j}=1 / 2$, then the random walk takes place on the $x$ axis, i.e., $\left(X_{n}, Y_{n}\right)$ reduces to a simple symmetric random walk on the real line. This case is excluded from this investigation. Hence the assumption that $\min _{j \in \mathbb{Z}} p_{j}<1 / 2$.

When $p_{j}=1 / 2, j= \pm 1, \pm 2, \ldots$, but $p_{0}=1 / 4$, then all the vertical lines $x=$ $j= \pm 1, \pm 2, \ldots$ are missing, except that of $x=0$, i.e., the $y$ axis. Thus we get what could be called a random walk on a "horizontal comb", whose vertical version was 
studied by Weiss and Havlin (1986), Bertacchi and Zucca (2003), Bertacchi (2006), Csáki et al. (2009, 2011).

Back to the random walk (1.1), even though the transition probabilities depend on the position of the first coordinate of $\mathbf{Z}_{n}:=\left(X_{n}, Y_{n}\right), n \in \mathbf{N}$, both $X_{n}$ and $Y_{n}$ will be seen to behave like a simple symmetric random walk on $\mathbb{Z}$, except for a random time delay, and independently of each other.

Heyde (1982) assumed the following asymptotic density condition for the transition probabilities of (1.1):

$$
k^{-1} \sum_{j=1}^{k} p_{j}^{-1}=2 \gamma+o\left(k^{-\eta}\right), \quad k^{-1} \sum_{j=1}^{k} p_{-j}^{-1}=2 \gamma+o\left(k^{-\eta}\right)
$$

as $k \rightarrow \infty$, for some constants $1<\gamma<\infty$ and $1 / 2<\eta<\infty$.

Under condition (1.2), for the first coordinate of $\mathbf{Z}_{n}$, Heyde (1982) concluded his Theorem 1 that reads as follows.

Theorem A On an appropriate probability space for $X_{n}$ there is a standard Wiener process $\{W(t), t \geq 0\}$ so that

$$
\gamma^{1 / 2} X_{n}=W\left(n\left(1+\varepsilon_{n}\right)\right)+O\left(n^{1 / 4}(\log n)^{1 / 2}(\log \log n)^{1 / 2}\right) \text { a.s. }
$$

as $n \rightarrow \infty$, with $\varepsilon_{n} \rightarrow 0$ a.s.

As to this theorem, Heyde (1982) writes:

"If strong additional assumptions are made about the column types, for example if there is a finite number of different types which occur with fixed periodicity, then the random variable $\varepsilon_{n}$ can safely be removed and relegated to the error in (1.3). We shall not pursue this question here because the presence of $\epsilon_{n}$ does not cause undue complication in the extraction of specific results from (1.3) as evidenced by Corollary 1 below."

The just mentioned Corollary 1 in Heyde (1982) reads as follows.

Corollary A Under condition (1.2), we have

(i) $\gamma^{1 / 2} n^{-1 / 2} X_{n} \rightarrow_{d} N(0,1)$, as $n \rightarrow \infty$,

(ii) $\lim \sup _{n \rightarrow \infty}\left(2 n \gamma^{-1} \log \log n\right)^{-1 / 2} X_{n}=1$ a.s. ,

(iii) $\liminf _{n \rightarrow \infty}\left(2 n \gamma^{-1} \log \log n\right)^{-1 / 2} X_{n}=-1$ a.s.

One of the aims of this exposition is to show that no additional conditions are needed for removing and relegating the random variable $\varepsilon_{n}$ to the error in (1.3) 
(cf. (1.13) of our Theorem 2, Corollary 2 and our conclusion right after the latter corollary). Let $\sigma_{0}=0<\sigma_{1}<\sigma_{2}<\cdots$ be the successive times at which the values of $X_{i}-X_{i-1}, i=1,2, \ldots$, are nonzero, and put $S_{1}(k)=X_{\sigma_{k}}$. By the assumed symmetry of the transition probabilities in (1.1), $S_{1}(k)$ is a simple symmetric random walk on $\mathbb{Z}$.

In view of this and (1.2), Heyde (1982) concludes his Lemma 1: $\sum_{1}^{\infty} n^{-2} E p_{S_{n}}^{-2}<$ $\infty$ and hence $\sum_{1}^{\infty} n^{-2} p_{S_{n}}^{-2}<\infty$ a.s. This, in turn, leads to the principal result that is needed for establishing his Theorem 1, i.e., Theorem A as above, namely to Theorem 2 of Heyde (1982):

$$
n^{-1} \sigma_{n} \rightarrow \gamma \text { a.s., as } n \rightarrow \infty \text {. }
$$

Also, $X_{n}=X_{\sigma_{k}}$ for $\sigma_{k} \leq n<\sigma_{k+1}$. As in the proof of Theorem 1 of Heyde (1982), for $n$ fixed let

$$
\sigma_{k(n)}:=\max \left[j: 1 \leq j \leq n, X_{j} \neq X_{j-1}\right]
$$

Then

$$
X_{n}=X_{\sigma_{k(n)}}=S_{1}(k(n))
$$

is the horizontal position of the walk $\mathbf{Z}_{n}=\left(X_{n}, Y_{n}\right)$ after $k(n)$ horizontal steps in the first $n$ steps of $\mathbf{Z}_{n}$ and, using (1.4), under condition (1.2) we have

$$
n^{-1} k(n) \rightarrow \gamma^{-1} \text { a.s., } \quad \text { as } n \rightarrow \infty
$$

(cf. Proof of Theorem 1 of Heyde (1982)). The latter conclusion in combination with Strassen's invariance principle (cf. Strassen (1967)) as in Heyde (1982), results in his Theorem 1 (cf. Theorem A above).

Clearly, in view of $(1.5), \ell(n):=n-k(n)$ is the number of vertical steps in the first $n$ steps of $\mathbf{Z}_{n}$ and, as a consequence of (1.6), under condition (1.2) we also have

$$
n^{-1} \ell(n) \rightarrow 1-\gamma^{-1} \text { a.s., as } n \rightarrow \infty \text {. }
$$

Under the weaker condition that $\eta=0$ in (1.2), Heyde (1993) concluded (1.6) and (1.7) in probability, i.e., that as $n \rightarrow \infty$, we have

$$
n^{-1} k(n) \rightarrow \gamma^{-1} \text { and } n^{-1} \ell(n) \rightarrow 1-\gamma^{-1} \text { in probability. }
$$

Using this together with the Cramér-Wold device in combination with the martingale limit result of Theorem 3.2 in Hall and Heyde (1980), Heyde (1993) established the following asymptotic joint distribution for the random walk $\mathbf{Z}_{n}=\left(X_{n}, Y_{n}\right)$.

Theorem B Assume (1.2) with $\eta=0$. Then, as $n \rightarrow \infty$,

$$
n^{-1 / 2} \mathbf{Z}_{n}=\left(\frac{X_{n}}{n^{1 / 2}}, \frac{Y_{n}}{n^{1 / 2}}\right) \rightarrow_{d}\left(\gamma^{-1 / 2} N_{1},\left(1-\gamma^{-1}\right)^{1 / 2} N_{2}\right)
$$


where $N_{1}$ and $N_{2}$ are independent standard normal random variables, and

$$
E X_{n}^{2} \sim \gamma^{-1} n \text { and } E Y_{n}^{2} \sim\left(1-\gamma^{-1}\right) n
$$

The symbol $\sim$ means that the ratio of the two sides tends to 1 as $n \rightarrow \infty$. Here, and throughout, $\rightarrow_{d}$ stands for convergence in distribution.

Thus, as noted by Heyde (1993), the asymptotic behaviour of $n^{-1 / 2}\left(X_{n}, Y_{n}\right)$ depends only on the macroscopic properties of the medium through which the walk takes place, i.e., of the $\left\{p_{j}, j \in \mathbb{Z}\right\}$ as in (1.2), with $\eta=0$ in this particular case. In the same paper Heyde also notes that this is of considerable practical significance since, although many materials are heterogeneous on a microscopic scale, they are essentially homogeneous on a macroscopic or laboratory scale. In conclusion Heyde (1993) writes (cf. 3. Final Remark):

"The so called dimensional anisotropy is $\left(1-\gamma^{-1}\right) / \gamma^{-1}$ and for a material like TTF-TCNQ we expect this to be, say $10^{-2}$. It should be noted that this would be achieved, for example, if only every 100th column were connective. We would have $p_{j}=\frac{1}{2}$ for $j$ a non-connective column and $p_{j}=\frac{1}{4}$ for $j$ a connective column."

Remark 1 We note that condition (1.2) clearly holds true if $\left\{p_{j}\right\}_{j \in \mathbb{Z}}$ is a periodic sequence. Namely, in this case, for a given positive integer $L \geq 1, p_{j+L}=p_{j}$ for all $j \in \mathbb{Z}$, and for $i=0,1, \ldots$, we have

$$
\frac{1}{L} \sum_{j=0}^{L-1} \frac{1}{p_{j+i L}}=\frac{1}{L} \sum_{j=0}^{L-1} \frac{1}{p_{j}}=2 \gamma,
$$

with some constant $1<\gamma<\infty$, as in (1.2). A particular periodic case, the so-called uniform case, when $p_{j}=1 / 4$ if $|j| \equiv 0(\bmod \mathrm{L})$ and $p_{j}=1 / 2$ otherwise yields (1.9) with $\gamma=(L+1) / L$. This uniform periodic case may serve as a model for describing dimensional anisotropies as in the just quoted example of Heyde (1993) via $\left(1-\gamma^{-1}\right) / \gamma^{-1}=\gamma-1=1 / L$.

Csáki et al. (2013) study the asymptotic behaviour of the anisotropic random walk $\left\{X_{n}, Y_{n}\right\}$ under both conditions (1.2) and (1.9).

In order to formulate our results here, consider $D\left([0, \infty), \mathbb{R}^{2}\right)$, the space of $\mathbb{R}^{2}$ valued càdlàg functions on $[0, \infty)$. For functions $(f(t), g(t))=\left(\left(f_{1}(t), f_{2}(t)\right)\right.$, $\left.\left(g_{1}(t), g_{2}(t)\right)\right)$ in this function space, define for all fixed $T>0$

$$
\Delta=\Delta_{T}(f, g):=\sup _{0 \leq t \leq T}\left\|\left(f_{1}(t)-g_{1}(t)\right),\left(f_{2}(t)-g_{2}(t)\right)\right\|,
$$

where $\|\cdot\|$ is a norm in $\mathbb{R}^{2}$, usually the $\|\cdot\|_{p}$ norm with $p=1$ or 2 in our case. 
Based on Heyde's result as in (1.8) that is implied by condition (1.2) with $\eta=0$, our first new conclusion is a Donsker type weak invariance principle, an extension of Heyde's bivariate central limit theorem (CLT) as in (a) of Theorem B, that reads as follows.

Theorem 1 With $X_{0}=Y_{0}=0$, let $\left\{X_{n}, Y_{n}, n \geq 0\right\}$ be the horizontal and vertical positions after $n$ steps of the random walk on $\mathbb{Z}^{2}$ with transition probabilities as in (1.1), and assume that condition (1.2) holds true with $\eta=0$. Then, on an appropriate probability space for this random walk $\mathbf{Z}_{n}:=\left\{X_{n}, Y_{n}\right\}$ on $\mathbb{Z}^{2}$, one can construct two independent standard Wiener processes $\left\{W_{1}(t), t \geq 0\right\},\left\{W_{2}(t), t \geq 0\right\}$ so that, as $n \rightarrow \infty$, with

$$
\left\{\mathbf{W}_{n}(t), t \geq 0\right\}_{n \geq 0}:=\left\{\frac{W_{1}\left(n t \gamma^{-1}\right)}{n^{1 / 2}}, \frac{W_{2}\left(n t\left(1-\gamma^{-1}\right)\right)}{n^{1 / 2}}, t \geq 0\right\}_{n \geq 0}
$$

we have for all fixed $T>0$

$$
\begin{aligned}
& \sup _{0 \leq t \leq T}\left\|n^{-1 / 2} \mathbf{Z}_{[n t]}-\mathbf{W}_{n}(t)\right\| \\
& =\sup _{0 \leq t \leq T}\left\|\frac{X_{[n t]}-W_{1}\left(n t \gamma^{-1}\right)}{n^{1 / 2}}, \frac{Y_{[n t]}-W_{2}\left(n t\left(1-\gamma^{-1}\right)\right)}{n^{1 / 2}}\right\| \\
& =o_{P}(1) .
\end{aligned}
$$

Moreover, based on (1.6) instead of (1.8) that is implied by assuming (1.2) as postulated with $1 / 2<\eta<\infty$, we arrive at a strong Strassen type invariance principle for $\mathbf{Z}_{n}:=\left\{X_{n}, Y_{n}\right\}$ on $\mathbb{Z}^{2}$ as follows.

Theorem 2 With $X_{0}=Y_{0}=0$, let $\left\{X_{n}, Y_{n}, n \geq 0\right\}$ be the horizontal and vertical positions after $n$ steps of the random walk on the integer lattice $\mathbb{Z}^{2}$ with transition probabilities as in (1.1) and assume that condition (1.2) holds true. Then, on an appropriate probability space for this random walk $\mathbf{Z}_{n}:=\left\{X_{n}, Y_{n}\right\}$ on $\mathbb{Z}^{2}$, one can construct two independent standard Wiener processes $\left\{W_{1}(t), t \geq 0\right\},\left\{W_{2}(t), t \geq 0\right\}$ so that, as $n \rightarrow \infty$, with $\left\{\mathbf{W}_{n}(t), t \geq 0\right\}_{n \geq 0}$ as in (1.11), we have

$$
\begin{aligned}
& \sup _{0 \leq t \leq 1}\left\|(2 n \log \log n)^{-1 / 2} \mathbf{Z}_{[n t]}-(2 \log \log n)^{-1 / 2} \mathbf{W}_{n}(t)\right\| \\
& =\sup _{0 \leq t \leq 1}\left\|\left(\frac{X_{[n t]}-W_{1}\left(n t \gamma^{-1}\right)}{(2 n \log \log n)^{1 / 2}}, \frac{Y_{[n t]}-W_{2}\left(n t\left(1-\gamma^{-1}\right)\right)}{(2 n \log \log n)^{1 / 2}}\right)\right\| \\
& =o(1) \text { a.s. }
\end{aligned}
$$


Remark 2 It will be seen when proving Theorems 1 and 2 that the respective conclusions of (1.12) and (1.13) hold simultaneously on the same probability space in terms of the there constructed two independent standard Wiener processes $W_{1}$ and $W_{2}$. Thus $\mathbf{W}_{n}$ in (1.13) coincides with that of (1.11) not only by notation, but also by construction.

Define now the measurable space $\left(D\left([0, \infty), \mathbb{R}^{2}\right), \mathcal{D}\right)$, where $\mathcal{D}$ is the $\sigma$-field generated by the collection of all $\Delta$-open balls for all $T>0$ of the function space $D\left([0, \infty), \mathbb{R}^{2}\right)$, where a ball is a subset of $D\left([0, \infty), \mathbb{R}^{2}\right)$ of the form

$$
\left\{\left(f_{1}, f_{2}\right): \Delta_{T}\left(\left(f_{1}, f_{2}\right),\left(g_{1}, g_{2}\right)\right)<r\right\}
$$

for some $\left(g_{1}, g_{2}\right) \in D\left([0, \infty), \mathbb{R}^{2}\right)$ and $r>0$, with $\Delta_{T}$ as in (1.10).

As a consequence of (1.12) of Theorem 1 , on taking $T=1$, we may, for example, conclude weak convergence for the càdlàg process $n^{-1 / 2} \mathbf{Z}_{[n \cdot]}$ in $D\left([0,1], \mathbb{R}^{2}\right)$ in terms of the following functional convergence in distribution statement.

Corollary 1 Under the conditions of Theorem 1 , as $n \rightarrow \infty$, we have

$$
\begin{aligned}
h\left(n^{-1 / 2} \mathbf{Z}_{[n t]}\right) & =h\left(\frac{X_{[n t]}}{n^{1 / 2}}, \frac{Y_{[n t]}}{n^{1 / 2}}\right) \rightarrow_{d} h\left(\mathbf{W}(t) \operatorname{diag}\left(\gamma^{-1 / 2},\left(1-\gamma^{-1}\right)^{1 / 2}\right)\right) \\
& \stackrel{d}{=} h\left(W_{1}\left(t \gamma^{-1}\right), W_{2}\left(t\left(1-\gamma^{-1}\right)\right)\right.
\end{aligned}
$$

for all $h: D\left([0,1], \mathbb{R}^{2}\right) \rightarrow \mathbb{R}^{2}$ that are $\left(D\left([0,1], \mathbb{R}^{2}\right), \mathcal{D}\right)$ measurable and $\Delta$-continuous, or $\Delta$-continuous except at points forming a set of measure zero on $\left(D\left([0,1], \mathbb{R}^{2}\right), \mathcal{D}\right)$ with respect to the Wiener measure $\mathbb{W}$ of $(\mathbf{W}(t), 0 \leq t \leq 1):=\left(\left(W_{1}(t), W_{2}(t)\right), 0 \leq\right.$ $t \leq 1)$, where $W_{1}$ and $W_{2}$ are two independent standard Wiener processes, and $\stackrel{d}{=}$ stands for equality in distribution.

The Brownian motion $\mathbf{W}(t) \operatorname{diag}\left(\gamma^{-1 / 2},\left(1-\gamma^{-1}\right)^{1 / 2}\right)$ on $\mathbb{R}^{2}$ with the indicated diffusion matrix $\operatorname{diag}(\cdot, \cdot)$ is an example of an anisotropic Brownian motion.

Remark 3 In the context of Corollary $1, \mathcal{D}$ of the measurable space $\left(D\left([0,1], \mathbb{R}^{2}\right), \mathcal{D}\right)$ coincides with the $\sigma$-field generated by the $\Delta$-open balls of the function space $D\left([0,1], \mathbb{R}^{2}\right)$ equipped with the norm $\Delta$ as in (1.8) with $T=1$. Clearly, the Brownian motion (Wiener process) $(\mathbf{W}(t), 0 \leq t \leq 1):=\left(W_{1}(t), W_{2}(t), 0 \leq t \leq 1\right)$, where $W_{1}$ and $W_{2}$ are two independent standard Wiener processes, is a random element of $\left(C\left([0,1], \mathbb{R}^{2}\right), \mathcal{E}\right)$, the measurable space of $\mathbb{R}^{2}$-valued continuous functions on $[0,1]$, where $\mathcal{E}$ is the Borel $\sigma$-field generated by the $\Delta$-open subsets of the space of $\mathbb{R}^{2}$-valued continuous functions $C\left([0,1], \mathbb{R}^{2}\right)$. Since

$$
C\left([0,1], \mathbb{R}^{2}\right) \in D\left([0,1], \mathbb{R}^{2}\right) \text { and } \mathcal{E}=C\left([0,1], \mathbb{R}^{2}\right) \cap \mathcal{D},
$$

$\mathbf{W}(t)$ is also a random element of $\left(D\left([0,1], \mathbb{R}^{2}\right), \mathcal{D}\right)$, and we can extend the Wiener measure $\mathbb{W}$ of $\{\mathbf{W}(t), 0 \leq t \leq 1\}$ on $\left(C\left([0,1], \mathbb{R}^{2}\right), \mathcal{E}\right)$ to $\left(D\left([0,1], \mathbb{R}^{2}\right), \mathcal{D}\right)$ as follows: 
for any set $A \in \mathcal{D}$, we have $A \cap C\left([0,1], \mathbb{R}^{2}\right)=: B \in \mathcal{E}$ and, via putting $\mathbb{W}(A)=\mathbb{W}(B)$ for $A \in \mathcal{D}$, we retain $\mathbb{W}\left(C\left([0,1], \mathbb{R}^{2}\right)\right)=1$. Also, on account of (1.15), for any map $h: D\left([0,1], \mathbb{R}^{2}\right) \rightarrow \mathbb{R}^{2}$ that is $\mathcal{D}$ measurable, with $h$ as in (1.14), the $\mathbb{R}^{2}$ valued map $h\left(\mathbf{W}(t) \operatorname{diag}\left(\gamma^{-1 / 2},\left(1-\gamma^{-1}\right)^{1 / 2}\right)\right)$ is seen to be a two dimensional random vector. For further details concerning the above lines, we refer to the middle three paragraphs of page 2948 of Csörgő and Martsynyuk (2011). Also, in Section 1.2 of the latter paper, we say that a sequence of random elements $\left\{G_{n}(t), 0 \leq t \leq 1\right\}_{n \geq 1}$ of $\left(D\left([0,1], \mathbb{R}^{2}\right), \mathcal{D}\right)$ converges weakly to a continuous random element $\{G(t), 0 \leq$ $t \leq 1\}$ of $\left(D\left([0,1], \mathbb{R}^{2}\right), \mathcal{D}\right)$, if, as $n \rightarrow \infty, g\left(G_{n}(t)\right) \rightarrow_{d} g(G(t))$ for all functionals $g: D\left([0,1], \mathbb{R}^{2}\right) \rightarrow \mathbb{R}$ that are $\mathcal{D}$ measurable and $\Delta$-continuous, or $\Delta$-continuous except on a set of measure zero in $\mathcal{D}$ with respect to the measure generated by $G(t)$. We note that Corollary 1 also holds true in terms of the latter definition of weak convergence, i.e., with replacing $G_{n}(t)$ by $n^{-1 / 2} \mathbf{Z}_{[n t]}$, and $G(t)$ by $\mathbf{W}(t)$, in this definition in hand (cf. also Remarks 1.2, 1.3, and Proposition 1.1. of Csörgö and Martsynyuk (2011)).

Back to Corollary 1, on taking $t=1$, it reduces to Theorem of Heyde (1993) (cf. Theorem B).

As another immediate example of Corollary 1 , as $n \rightarrow \infty$, under the conditions of Theorem 1, we conclude

$$
\begin{aligned}
& \left(n^{-1 / 2} \int_{0}^{1} X_{[n t]} d t, n^{-1 / 2} \int_{0}^{1} Y_{[n t]} d t\right) \\
& \rightarrow_{d}\left(\gamma^{-1 / 2} \int_{0}^{1} W_{1}(t) d t,\left(1-\gamma^{-1}\right)^{1 / 2} \int_{0}^{1} W_{2}(t) d t\right) \\
& \quad \stackrel{d}{=}\left(\gamma^{-1 / 2} N_{1}(0,1 / 3),\left(1-\gamma^{-1}\right)^{1 / 2} N_{2}(0,1 / 3)\right),
\end{aligned}
$$

where $N_{1}(0,1 / 3)$ and $N_{2}(0,1 / 3)$ are independent normal random variables with mean 0 and variance $1 / 3$. Here, and throughout, $\stackrel{d}{=}$ stands for equality in a distribution.

Define now the continuous time process $\mathbf{Z}_{n t}:=\left\{X_{n t}, Y_{n t}\right\}$ by linear interpolation for $t \in[0,1]$, i.e., $\mathbf{Z}_{n t}$ are random elements of $C\left([0,1], \mathbb{R}^{2}\right)$, and interpret the uniform $\Delta$-norm defined in (1.10) for functions in this function space.

Recall the definition of the two dimensional Strassen (1964) class of absolutely continuous functions:

$$
\mathcal{S}^{(2)}=\left\{(f(x), g(x)), 0 \leq x \leq 1: f(0)=g(0)=0, \int_{0}^{1}\left(\dot{f}^{2}(x)+\dot{g}^{2}(x)\right) d x \leq 1\right\} .
$$

As noted already in the Introduction, via using an alternative construction of random walk $\left\{X_{n}, Y_{n}\right\}$ on $\mathbb{Z}^{2}$ with horizontal step transition probabilities $1 / 2-p_{j}$ 
and vertical step transition probabilities $p_{j}$, i.e., an inverted version of transition probabilities as in (1.1), and assuming condition (1.2), Csáki et al. (2013) established a joint strong approximation for $\left\{X_{n}, Y_{n}\right\}$ by two independent standard Wiener processes as in their Theorem 1.1. As a consequence of the latter Theorem 1.1 and the two-dimensional version of the Strassen (1964) functional iterated logarithm law that is stated as Lemma D in Csáki et al. (2013) for the continuous time process $\mathbf{Z}_{n t}:=\left\{X_{n t}, Y_{n t}\right\}$ as right above, they concluded a functional law of the iterated logarithm as stated in their Corollary 1.1. Via our Theorem 2 parts (i)-(iii) of the latter corollary are inherited in the present context, and they read as follows.

Corollary $\mathbf{2}$ For the random walk $\mathbf{Z}_{n}$, as a consequence of Theorem 2, we conclude that

(i) the sequence of random vector-valued functions

$$
\left(\gamma^{1 / 2} \frac{X_{n x}}{(2 n \log \log n)^{1 / 2}},\left(\frac{\gamma}{\gamma-1}\right)^{1 / 2} \frac{Y_{n x}}{(2 n \log \log n)^{1 / 2}}, 0 \leq x \leq 1\right)_{n \geq 3}
$$

is almost surely relatively compact in $C\left([0,1], \mathbb{R}^{2}\right)$ in the uniform $\Delta$-norm topology, and its limit points is the set of functions $\mathcal{S}^{(2)}$ (i.e., the collection of a.s. limits of convergent subsequences in uniform $\Delta$-norm).

(ii) In particular, the vector sequence

$$
\left(\frac{X_{n}}{(2 n \log \log n)^{1 / 2}} \frac{Y_{n}}{(2 n \log \log n)^{1 / 2}}\right)_{n \geq 3}
$$

is almost surely relatively compact in the rectangle

$$
\left[-\frac{1}{\sqrt{\gamma}}, \frac{1}{\sqrt{\gamma}}\right] \times\left[-\frac{\sqrt{\gamma-1}}{\sqrt{\gamma}}, \frac{\sqrt{\gamma-1}}{\sqrt{\gamma}}\right]
$$

and the set of its limit points is the ellipse

$$
\left\{(x, y): \gamma x^{2}+\frac{\gamma}{\gamma-1} y^{2} \leq 1\right\}
$$

(iii) Moreover,

$$
\limsup _{n \rightarrow \infty} \frac{X_{n}}{\sqrt{2 n \log \log n}}=\frac{1}{\sqrt{\gamma}} \text { a.s. }
$$

and

$$
\limsup _{n \rightarrow \infty} \frac{Y_{n}}{\sqrt{2 n \log \log n}}=\frac{\sqrt{\gamma-1}}{\sqrt{\gamma}} \text { a.s. }
$$

For a survey on the path behaviour of random walks on a 2-dimensional anisotropic lattice with asymptotic density conditions à la Heyde $(1982,1993)$ we refer to Csáki et al. (2015). 


\subsection{A nearest-neighbour random walk in a random environ- ment on $\mathbb{Z}^{2}$; Random column configurations}

Shuler (1979) formulated three conjectures on the asymptotic properties of a nearestneighbour random walk on $\mathbb{Z}^{2}$ that is allowed to make horizontal steps everywhere but vertical steps only on a random fraction of the columns.

Following den Hollander (1994), let

$$
C=\{C(x)\}_{x \in \mathbb{Z}}
$$

be a random $\{0,1\}$-valued sequence with probability law $\mu$ on $\{0,1\}^{\mathbb{Z}}$, satisfying the assumptions:

(*) $\quad \mu$ is stationary and ergodic (w.r.t. translations in $\mathbb{Z}$ ),

$$
0<q:=\mu(C(0)=1) \leq 1
$$

i.e., $q=E_{\mu} C(0)$, the expected value of $C(0)$ with respect to $\mu$.

We note in passing that due to the assumed stationarity of the probability measure $\mu$ on $\{0,1\}^{\mathbb{Z}}$, we have (1.17) for $C(x)$ for all $x \in \mathbb{Z}$ and $E_{\mu} C(x)=q$.

Given the sequence $C=\{C(x)\}_{x \in \mathbb{Z}}$, a random environment is constructed the following way: (i) the horizontal edges of the lattice $\mathbb{Z}^{2}$ are left unchanged, i.e., all the horizontal edges in all the rows are kept, and (ii) all the vertical edges in the column $x$ are left or erased, depending on whether $C(x)=1$ or $C(x)=0$. Thus, given $C=\{C(x)\}_{x \in \mathbb{Z}}$, all the rows are connected, but only a part of the columns are.

Now, given $C=\{C(x)\}_{x \in \mathbb{Z}}$, let

$$
\{\mathbf{Z}(n)\}_{n \geq 0}=\{X(n), Y(n)\}_{n \geq 0}
$$

be the random walk that starts from the origin at time 0 and chooses with equal probability one of the unerased edges of the lattice $\mathbb{Z}^{2}$ adjacent to the current position and jumps along it to a neighbouring site, i.e., given $C$, the random walk $\mathbf{Z}(n)$, $n \in \mathbf{N}$, on the path space $\left(\mathbb{Z}^{2}\right)^{\mathbf{N}}$ with probability law $P_{C}$ as in (4) of den Hollander (1994) has the following transition probabilities:

$$
\begin{aligned}
& P_{C}(\mathbf{Z}(n+1)=(x \pm 1, y) \mid \mathbf{Z}(n)=(x, y))=1 / 2 \quad \text { if } \quad C(x)=0 \\
& P_{C}(\mathbf{Z}(n+1)=(x \pm 1, y \pm 1) \mid \mathbf{Z}(n)=(x, y))=1 / 4 \quad \text { if } C(x)=1
\end{aligned}
$$

On integrating over $C$ with respect to $\mu$, the thus obtained random walk in random environment process has probability law $P:=\int P_{C} \mu(d C)$.

We note in passing that with $q=1,(1.19)$ becomes a simple symmetric random walk on $\mathbb{Z}^{2}$. 
The three conjectures formulated by Shuler (1979), and referred to as "Ansätze" by him, relate to the asymptotic behaviour of $\mathbf{Z}(n)$ under the law $P$ as $n \rightarrow \infty$. They concern the total number of steps and the mean-square displacement in the $x$ (horizontal) and $y$ (vertical) directions, the probability of return to the origin, and the expected number of distinct sites visited, in the first $n$ steps of $\mathbf{Z}(n)$.

For $n$ fixed, and keeping the notation used by den Hollander (1994), let

$$
\begin{aligned}
& n_{x}(n):=|\{0 \leq m<n: X(m+1) \neq X(m)\}|, \\
& n_{y}(n):=|\{0 \leq m<n: Y(m+1) \neq Y(m)\}|,
\end{aligned}
$$

respectively denote the total number of horizontal and vertical steps in the first $n$ steps of $\mathbf{Z}(n)$. Proved as Ansatz 1 of Shuler (1979) in den Hollander (1994), with $C=\{C(x)\}_{x \in \mathbb{Z}}$ of (1.16) and $\mu$ as in (1.17), we have

$$
\begin{aligned}
& \lim _{n \rightarrow \infty} \frac{n_{x}(n)}{n}=q_{x} \quad P \text {-a.s. } \\
& \lim _{n \rightarrow \infty} \frac{n_{y}(n)}{n}=q_{y} \quad P \text {-a.s. }
\end{aligned}
$$

Clearly, $n_{x}(n)+n_{y}(n)=n$ and, in view of (1.21), $q_{x}+q_{y}=1$. Also, as noted by den Hollander (1994), $q_{x}$ and $q_{y}$ denote the density of horizontal and vertical bonds in the lattice $\mathbb{Z}^{2}$, i.e., $q_{x}+q_{y}=1$ and $q_{y} / q_{x}=q$ by $(*)$. Consequently, the respective limits in (1.21) are

$$
q_{x}=1 /(1+q) \text { and } q_{y}=q /(1+q) .
$$

We note in passing that the $P$-a.s. conclusions of (1.21) can be viewed as respective analogues of those of (1.6) and (1.7) for the anisotropic random walk $\mathbf{Z}_{n}$ under the asymptotic density condition (1.2) for its transition probabilities as in (1.1) in that, under $(*)$, the asymptotic behaviour of the random walk $\mathbf{Z}(n)$ with its $P_{C}$ transition probabilities as in (1.19) integrated over $C$ behaves like an anisotropic random walk on $\mathbb{Z}^{2}$, with the anisotropy determined only by $q$ via $\mu$ of $(*)$. Moreover, $q$ viewed as the density of the connected columns via $q=q_{y} / q_{x}$, it "coincides" with the so-called dimensional anisotropy (cf. the quoted 3. Final Remark of Heyde (1993) right above Remark 1)

$$
\lim _{n \rightarrow \infty} \frac{n^{-1} \ell(n)}{n^{-1} k(n)}=\frac{1-\gamma^{-1}}{\gamma^{-1}}=\gamma-1
$$

of the anisotropic random walk $\mathbf{Z}_{n}$ under the condition (1.2) that, in turn, implies the respective a.s. conclusions of (1.6) and (1.7) via the Proof of Theorem 1 of Heyde (1982), as noted right after (1.6).

Remark 4 In view of our conclusion in Remark 1 concerning the so-called uniform periodic case, and our lines right above on $q=q_{y} / q_{x}$ versus (1.23), it appears to 
be reasonable to say that $(*)$ together with $(1.19)$, in the long run amount to a stationary ergodic randomization of deleting columns in the uniform periodic case on an average $q=E_{\mu} C(0)=E C(x)$ times, instead of $(\gamma-1)$ fraction of times as in (1.23) that, for a given positive integer $L \geq 1$, is equal to $1 / L$ in the fixed column uniform periodic configuration case. On taking $q=1 / L$ in $(*)$ in combination with the transition probabilities of (1.19) in the random column configuration model, in the long run amounts to realizing the dimensional anisotropy of a fixed column uniform periodic configuration $1 / L$ times on an average, instead of having it equal to $(\gamma-1)=1 / L$ eventually under the condition $(1.2)$.

Further along these lines, from the $P$-a.s. limits of $(1.21)$ that coincide with Ansatz 1 of den Hollander (1994), he concludes

$$
\begin{aligned}
& E X^{2}(n) \sim \frac{1}{1+q} n \\
& E Y^{2}(n) \sim \frac{q}{1+q} n .
\end{aligned}
$$

Here $E$ denotes expectation with respect to $P=\int P_{C} \mu(d C)$, and the statements themselves are analogous to (b) of Theorem B, established by Heyde (1993) under his condition (1.2) with $\eta=0$.

Under the additional mixing assumption (19) of den Hollander (1994) on $\mu$, he concludes

$$
Q(n):=P(\mathbf{Z}(2 n)=(0,0)) \sim \frac{1+q}{2 \pi q^{1 / 2} n}
$$

and

$$
R(n):=E|\{\mathbf{Z}(0), \mathbf{Z}(1), \ldots, \mathbf{Z}(n)\}| \sim \frac{2 \pi q^{1 / 2} n}{(1+q) \log n},
$$

where $E$ denotes expectation with respect to $P$ as in (1.24), and the integrand $|\{\mathbf{Z}(0), \mathbf{Z}(1), \ldots, \mathbf{Z}(n)\}|$ stands for the number of distinct sites visited, as in den Hollander (1994).

As noted before, with $q=1,(1.19)$ becomes a simple symmetric random walk on $\mathbb{Z}^{2}$, and (1.25) and (1.26) are well known results in this classical context.

Also, as noted already, the conclusions of (1.21) constitute Ansatz 1 of Shuler (1979). We also note in passing that the respective results of (1.25) and (1.26) that are based on those of (1.24), that is (10) in den Hollander (1994), coincide with (11) and (12) of den Hollander (1994), which in turn respectively amount to Ansatz 2 and Ansatz 3 of Shuler (1979). Concerning the latter (10), (11) and (12) of his paper, den Hollander (1994) writes as follows:

"Note that the coefficients in (10)-(12) depend on $\mu$ only via the density of connected columns q. Moreover, (10)-(12) are precisely what one 
would find for a random walk on the full lattice that makes horizontal and vertical steps with probability $q_{x}=1 /(1+q)$, resp. $q_{y}=q /(1+q)$. Actually, this was the main idea behind the formulation of the Ansätze in the first place: asymptotically the random walk should behave like an anisotropic random walk on $\mathbb{Z}^{2}$, with the anisotropy determined only by $q$ and not by any other parameters of $\mu$. Though, as we shall see later, this is not quite true in full generality under $(*)$ [not for $Q(n)$ and $R(n)$ at least], it is indeed true for many asymptotic properties associated with the random walk and within a large class of column distributions (including periodic distributions and i.i.d. distributions)."

The in here mentioned condition $(*)$ of den Hollander (1994) is that of (1.17) on $\mu$ above, and the hinted at further condition is the mixing assumption of (19) on $\mu$ in den Hollander (1994) that we already mentioned fleetingly when introducing (1.25) and (1.26) above.

Observation In this section we are to spell out analogues of Theorems 1 and 2 under the $(*)$ den Hollander (1994) condition of (1.17) on $\mu$. These analogues will constitute evidence to saying that, under the latter condition, asymptotically the random walk $\mathbf{Z}(n)$ in random environment with probability law $P=\int P_{C} \mu(d C)$ (cf. (1.16), (1.19) respectively for $C$ and $P_{C}$ ) behaves like an anisotropic random walk on $\mathbb{Z}^{2}$ does, with the anisotropy determined only by $q$ and not by any other parameters of $\mu$, as if it were an anisotropic 2-dimensional random walk under the asymptotic density condition of Heyde (1982), with $\gamma=1+q, 0<q \leq 1$, in (1.2) for the transition probabilities of (1.1).

As in den Hollander (1994), let $B_{q}=\left\{B_{q}(t)\right\}_{t \geq 0}$ be anisotropic Brownian motion on $\mathbb{R}^{2}$ with diffusion matrix

$$
D=\left(\begin{array}{cc}
1 /(1+q) & 0 \\
0 & q /(1+q)
\end{array}\right),
$$

i.e., $E B_{q}(t)=(0,0)$ and the finite dimensional distributions of $\left\{B_{q}(t)\right\}_{t>0}$ are Gaussian with covariance matrix $\min (s, t) D, 0 \leq s, t<\infty$. Consequently, $\left\{B_{q}(t)\right\}_{t \geq 0}$ has the following representation

$$
\begin{aligned}
\left\{B_{q}(t)\right\}_{t \geq 0} & \stackrel{d}{=}\left\{\mathbf{W}(t) \operatorname{diag}\left((1 /(1+q))^{1 / 2},(q /(1+q))^{1 / 2}\right)\right\}_{t \geq 0} \\
& \stackrel{d}{=}\left\{W_{1}(t /(1+q)), W_{2}(t q /(1+q))\right\}_{t \geq 0}
\end{aligned}
$$

with $\{\mathbf{W}(t)\}_{t \geq 0}:=\left\{\left(W_{1}(t), W_{2}(t)\right)\right\}_{t \geq 0}$, where $\left\{W_{1}(t), t \geq 0\right\}$ and $\left\{W_{2}(t), t \geq 0\right\}$ are two independent standard Wiener processes.

We now reformulate the invariance principle of Theorem 1 of den Hollander (1994) for $\mathbf{Z}(n)$ à la Theorem 1 in our previous section. 
Theorem C Assume $(*)$ for the probability law $\mu$ of the random $\{0,1\}$-valued sequence $\{C(x)\}_{x \in \mathbb{Z}}$ on $\{0,1\}^{\mathbb{Z}}$. Then (1.21) is true and, on an appropriate probability space for $\mathbf{Z}(n)$ on $\mathbb{Z}^{2}$ with transition probabilities as in (1.19) and probability law $P:=\int P_{C} \mu(d C)$, one can construct two independent standard Wiener processes $\left\{W_{1}(t), t \geq 0\right\},\left\{W_{2}(t), t \geq 0\right\}$ so that, as $n \rightarrow \infty$, with

$$
\left\{\mathbf{W}_{n}(t), t \geq 0\right\}_{n \geq 0}:=\left\{\frac{W_{1}(n t /(1+q))}{n^{1 / 2}}, \frac{W_{2}(n t q /(1+q))}{n^{1 / 2}}, t \geq 0\right\}_{n \geq 0}
$$

and $q$ as in (1.17), we have for all fixed $T>0$

$$
\begin{aligned}
& \sup _{0 \leq t \leq T}\left\|n^{-1 / 2} \mathbf{Z}([n t])-\mathbf{W}_{n}(t)\right\| \\
& =\sup _{0 \leq t \leq T}\left\|\frac{X([n t])-W_{1}(n t /(1+q))}{n^{1 / 2}}, \frac{Y([n t])-W_{2}(n t q /(1+q))}{n^{1 / 2}}\right\| \\
& =o_{P}(1) .
\end{aligned}
$$

Moreover, under the same condition, i.e., only under the $(*)$ den Hollander (1994) condition of (1.17) on $\mu$, we also have the following Strassen type extended version of (21) of his Theorem 2 (ibid.).

Theorem 3 Assume $(*)$ as in (1.17). Then on the probability space of Theorem $\mathrm{C}$ with the same independent standard Wiener processes $W_{1}$ and $W_{2}$, we have

$$
\begin{aligned}
& \sup _{0 \leq t \leq 1}\left\|(2 n \log \log n)^{-1 / 2} \mathbf{Z}([n t])-(2 \log \log n)^{-1 / 2} \mathbf{W}_{n}(t)\right\| \\
& =\sup _{0 \leq t \leq 1}\left\|\frac{X([n t])-W_{1}(n t /(1+q))}{(2 n \log \log n)^{1 / 2}}, \frac{Y([n t])-W_{2}(n t q /(1+q))}{(2 n \log \log n)^{1 / 2}}\right\| \\
& =o(1) \quad P \text {-a.s., } n \rightarrow \infty .
\end{aligned}
$$

Remark 5 Under their respective conditions, Theorem 1 and Theorem C "coincide", and so do also Theorem 2 and Theorem 3. Thus, to the extent of simultaneously having a weak Donsker and a strong Strassen type asymptotic behaviour, the two random walks in hand behave similarly. Moreover, just like having Theorem 1 under the weaker conditions of (1.8) than that of (1.6), Theorem $\mathrm{C}$ will also be seen to be true under assuming only convergence in probability versions of the $P$-a.s. conclusions of (1.21). We do not however know how to go about weakening the $(*)$ assumption of (1.17) for the probability law $\mu$ so that it would only yield the desired weaker version of (1.21).

Remark 6 Mutatis mutandis, Corollaries 1 and 2 also hold true in the context of Theorems $\mathrm{C}$ and 3 respectively. 


\section{Proofs}

\subsection{Preliminaries and proofs of Theorems 1 and 2}

As right after Corollary $\mathrm{A} \grave{a}$ la 3 . Proofs of Heyde (1982), let again $\sigma_{0}=0<\sigma_{1}<$ $\sigma_{2}<\cdots$ be the successive times at which the values of the $X_{i}-X_{i-1}, i=1,2, \ldots$ are nonzero, and put again $S_{1}(k)=X_{\sigma_{k}}$. By the assumed symmetry of the transition probabilities in (1.1), $\left\{S_{1}(k), k \geq 0\right\}$ is a simple symmetric random walk on $\mathbb{Z}$. Also, $X_{n}=X_{\sigma_{k}}$ for $\sigma_{k} \leq n<\sigma_{k+1}$. For $n$ fixed let

$$
\sigma_{k(n)}:=\max \left[j: j \leq n, X_{j} \neq X_{j-1}\right]
$$

Then again, as in (1.5),

$$
X_{n}=X_{\sigma_{k(n)}}=S_{1}(k(n))
$$

is the horizontal position of the walk $\mathbf{Z}_{n}=\left(X_{n}, Y_{n}\right)$ after $k(n)$ horizontal steps in the first $n$ steps of $\mathbf{Z}_{n}$.

Clearly, in view of $(2.1), \ell(n):=n-k(n)$ is the number of vertical steps in the first $n$ steps of $\mathbf{Z}_{n}$. On its own, the vertical position $Y_{n}$ of the walk $\mathbf{Z}_{n}=\left(X_{n}, Y_{n}\right)$ can be dealt with similarly to that of its horizontal position $X_{n}$. As in the proof of Theorem in Heyde (1993), let $\tau_{0}=0<\tau_{1}<\tau_{2}<\cdots$ be the successive times at which the values of $Y_{i}-Y_{i-1}, i=1,2, \ldots$ are nonzero and put $S_{2}(k)=Y_{\tau_{k}}$. Then, again by the assumed symmetry of the transition probabilities (1.1), $\left\{S_{2}(k), k \geq 0\right\}$ is a simple symmetric random walk on $\mathbb{Z}$, and $Y_{n}=Y_{\tau_{k}}$ for $\tau_{k} \leq n<\tau_{k+1}$. For $n$ fixed, let

$$
\tau_{\ell(n)}:=\max \left[j: j \leq n, Y_{j} \neq Y_{j-1}\right] .
$$

Then, for $Y_{n}$, the vertical position of the random walk $\mathbf{Z}_{n}=\left(X_{n}, Y_{n}\right)$ after $n$ steps, we have

$$
Y_{n}=Y_{\tau_{\ell(n)}}=S_{2}(\ell(n))
$$

after $\ell(n)$ vertical steps in the first $n$ steps of $\mathbf{Z}_{n}$.

As above, with $\sigma_{k}$ and $\tau_{k}$ standing for the times at which $X$ and $Y$ make their respective $k$-th steps $\left(\sigma_{0}=\tau_{0}=0\right)$, both

$$
\begin{array}{r}
\left\{X_{\sigma_{k}}, k \geq 0\right\}=\left\{S_{1}(k), k \geq 0\right\} \\
\left\{Y_{\tau_{k}}, k \geq 0\right\}=\left\{S_{2}(k), k \geq 0\right\}
\end{array}
$$

are simple symmetric random walks on $\mathbb{Z}$, and are defined independently of each other. Consequently, the random walk $\mathbf{Z}_{n}=\left(X_{n}, Y_{n}\right)_{n \geq 0}$ with transition probabilities as in (1.1) and viewed $\grave{a} l a(2.1)$ and (2.2) can be studied in terms of

$$
\mathbf{Z}_{n}=\left(X_{n}, Y_{n}\right)=\left(X_{\sigma_{k(n)}}, Y_{\tau_{\ell(n)}}\right)=\left(S_{1}(k(n)), S_{2}(\ell(n))\right.
$$


where, as before, $k(n)$ and $\ell(n)$ are the respective numbers of horizontal and vertical steps in the first $n$ steps of $\mathbf{Z}_{n}, S_{1}(\cdot)$ and $S_{2}(\cdot)$ are independent simple symmetric random walks on $\mathbb{Z}$, and $k(n)+\ell(n)=n$.

Now, without changing their distribution, on an appropriate probability space for the two independent simple symmetric random walks $\left\{S_{i}(j), j \geq 0\right\}, i=1,2$, one can construct two independent standard Wiener processes $\left\{W_{1}(t), t \geq 0\right\}$ and $\left\{W_{2}(t), t \geq 0\right\}$ so that (cf. Komlós, Major and Tusnády [KMT] (1975))

$$
\left|S_{i}(j)-W_{i}(j)\right|=O(\log j) \text { a.s., } i=1,2,
$$

as $j \rightarrow \infty$, and

$$
\sup _{0 \leq t \leq 1}\left|S_{i}([n t])-W_{i}(n t)\right|=O(\log n) \text { a.s., } i=1,2,
$$

as $n \rightarrow \infty$.

Consequently, as $n \rightarrow \infty$, the components of $\mathbf{Z}_{n}$ as in (2.5) can be studied independently via the approximations

$$
\begin{gathered}
\left|S_{1}(k(n))-W_{1}(k(n))\right|=O(\log k(n)) \text { a.s., } \\
\sup _{0 \leq t \leq 1}\left|S_{1}(k(n t))-W_{1}(k(n t))\right|=O(\log k(n)) \text { a.s., }
\end{gathered}
$$

and

$$
\begin{gathered}
\left|S_{2}(\ell(n))-W_{2}(\ell(n))\right|=O(\log \ell(n)) \text { a.s., } \\
\sup _{0 \leq t \leq 1}\left|S_{2}(\ell(n t))-W_{2}(\ell(n t))\right|=O(\log \ell(n)) \text { a.s. }
\end{gathered}
$$

where $k(n t)$ and $\ell(n t)$ are the respective numbers of horizontal and vertical steps in the first $[n t]$ steps of $\mathbf{Z}_{[n t]}, 0 \leq t \leq 1$, and $k(n t)+\ell(n t)=[n t]$.

Now, under condition (1.2) we have (1.6) and, as in the Proof of Theorem 1 of Heyde (1982), on writing $k(n)=n \gamma^{-1}\left(1+\varepsilon_{n}\right)$, with $\varepsilon_{n} \rightarrow 0$ a.s. as $n \rightarrow \infty$, we arrive at (cf. (2.8))

$$
\begin{aligned}
\left|S_{1}(k(n))-W_{1}\left(n \gamma^{-1}\left(1+\varepsilon_{n}\right)\right)\right| & =O\left(\log n \gamma^{-1}\left(1+\varepsilon_{n}\right)\right) \text { a.s. } \\
& =O(\log n) \text { a.s. }
\end{aligned}
$$

as $n \rightarrow \infty$. Moreover, as $n \rightarrow \infty$, we have also (cf. (2.9))

$$
\sup _{0 \leq t \leq 1}\left|S_{1}(k(n t))-W_{1}\left(n t \gamma^{-1}\left(1+\varepsilon_{n}\right)\right)\right|=O(\log n) \text { a.s. }
$$

Recalling that for $X_{n}$, the horizontal position of the random walk after $n$ steps, we have (cf. (2.1)) $X_{n}=X_{\sigma_{k(n)}}=S_{1}(k(n))$, via $(2.12)$, as $n \rightarrow \infty$, we arrive at

$$
\gamma^{1 / 2} X_{n}=W_{1}\left(n\left(1+\varepsilon_{n}\right)\right)+O(\log n) \text { a.s. }
$$


that in turn rhymes with (1.3) of Theorem A, a result of Heyde (1982), except the a.s. $O(\cdot)$ rate, that in his case is due to using a result of Strassen (1967), that amounts to the best possible approximation of the partial sums $S_{1}(\cdot)$ by Brownian motion via Skorokhod stopping times, while that of KMT (1975) that we use amounts to its globally best possible approximation. As we will however see later on, both serve equally well in the present context.

In view of (2.13), as $n \rightarrow \infty$, we have

$$
\sup _{0 \leq t \leq 1}\left|\frac{X_{[n t]}}{n^{1 / 2}}-\frac{W_{1}\left(n t \gamma^{-1}\left(1+\varepsilon_{n}\right)\right)}{n^{1 / 2}}\right|=O\left(\frac{\log n}{n^{1 / 2}}\right) \quad \text { a.s. }
$$

As to $Y_{n}$, the vertical position of the random walk $\mathbf{Z}_{n}=\left(X_{n}, Y_{n}\right)$, recall (cf. (2.5)) that we have $Y_{n}=Y_{\tau_{\ell(n)}}=S_{2}(\ell(n))$, as well as $\mathbf{Z}_{n}=\left(X_{n}, Y_{n}\right)=\left(S_{1}(k(n)), S_{2}(\ell(n))\right)$, and $k(n)+\ell(n)=n$, the sum of the number of horizontal and vertical steps in the first $n$ steps of $\mathbf{Z}_{n}$. Hence, in view of (1.7), and on writing $\ell(n)=n\left(1-\gamma^{-1}\right)\left(1+\tilde{\varepsilon}_{n}\right)$, with $\tilde{\varepsilon}_{n} \rightarrow 0$ a.s. as $n \rightarrow \infty$, mutatis mutandis in concluding (2.14), with a standard Wiener process $\left\{W_{2}(t), t \geq 0\right\}$ as in (2.6), we arrive at

$$
\sup _{0 \leq t \leq 1}\left|\frac{Y_{[n t]}}{n^{1 / 2}}-\frac{W_{2}\left(n t\left(1-\gamma^{-1}\right)\left(1+\tilde{\varepsilon}_{n}\right)\right)}{n^{1 / 2}}\right|=O\left(\frac{\log n}{n^{1 / 2}}\right) \quad \text { a.s. }
$$

as $n \rightarrow \infty$.

We note again that the conclusions of (2.12)-(2.15) are based on the Heyde (1982) condition (1.2) yielding (1.6) and (1.7), and, consequently, on writing

$$
k(n)=n \gamma^{-1}\left(1+\varepsilon_{n}\right), \ell(n)=n\left(1-\gamma^{-1}\right)\left(1+\tilde{\varepsilon}_{n}\right)
$$

with $\varepsilon_{n}$ and $\tilde{\varepsilon}_{n}$ both converging almost surely to 0 as $n \rightarrow \infty$.

We now recall that under the weaker condition that $\eta=0$ in (1.2), Heyde (1993) concluded (1.6) and (1.7) to hold true in probability as indicated in (1.8). Consequently, we can again write $k(n)$ and $\ell(n)$ as above in $(2.16)$, but now with $\varepsilon_{n}$ and $\tilde{\varepsilon}_{n}$ both converging in probability to 0 . This, in turn, results in having (2.12)(2.15) in probability instead of almost surely. In particular, for further use, we spell out the in probability versions of (2.14) and (2.15) as follows.

With the two independent standard Wiener processes $W_{1}$ and $W_{2}$ as in (2.6), and writing $k(n)$ and $\ell(n)$ as in (2.16) with $\varepsilon_{n}$ and $\tilde{\varepsilon}_{n}$ both converging in probability to 0 as $n \rightarrow \infty$ on account of having (1.8) under the condition that $\eta=0$ in (1.2), as $n \rightarrow \infty$, we arrive at

$$
\sup _{0 \leq t \leq 1}\left|\frac{X_{[n t]}}{n^{1 / 2}}-\frac{W_{1}\left(n t \gamma^{-1}\left(1+\varepsilon_{n}\right)\right)}{n^{1 / 2}}\right|=o_{P}(1)
$$

and

$$
\sup _{0 \leq t \leq 1}\left|\frac{Y_{[n t]}}{n^{1 / 2}}-\frac{W_{2}\left(n t\left(1-\gamma^{-1}\right)\left(1+\tilde{\varepsilon}_{n}\right)\right)}{n^{1 / 2}}\right|=o_{P}(1) .
$$


Lemma 1 Under the condition (1.2) as is, that via (2.16) yields the a.s. convergence of both $\varepsilon_{n}$ and $\tilde{\varepsilon}_{n}$ to 0 as $n \rightarrow \infty$, and also under the condition (1.2) with $\eta=0$, that via (2.16) results in $\varepsilon_{n}$ and $\tilde{\varepsilon}_{n}$ both converging in probability to 0 as $n \rightarrow \infty$, we have

$$
\sup _{0 \leq t \leq 1}\left|\frac{W_{1}\left(n t \gamma^{-1}\left(1+\varepsilon_{n}\right)\right)-W_{1}\left(n t \gamma^{-1}\right)}{n^{1 / 2}}\right|=o_{P}(1)
$$

as well as

$$
\sup _{0 \leq t \leq 1}\left|\frac{W_{2}\left(n t\left(1-\gamma^{-1}\right)\left(1+\tilde{\varepsilon}_{n}\right)\right)-W_{2}\left(n t\left(1-\gamma^{-1}\right)\right)}{n^{1 / 2}}\right|=o_{P}(1) .
$$

We note in passing that these two statements hold true in terms of any generic standard Wiener process $W$. The present forms are only for the sake of easy reference when proving Theorem 1, and, later on, when indicating the proof of Theorem $\mathrm{C}$.

Proof of Lemma 1 First assume (1.2). Then with $\varepsilon_{n} \rightarrow 0$ a.s. as $n \rightarrow \infty$, we have

$$
\begin{aligned}
& \sup _{0 \leq t \leq 1}\left|\frac{W_{1}\left(n t \gamma^{-1}\left(1+\varepsilon_{n}\right)\right)-W_{1}\left(n t \gamma^{-1}\right)}{n^{1 / 2}}\right| \\
& \stackrel{d}{=} \sup _{0 \leq t \leq 1}\left|W_{1}\left(t \gamma^{-1}\left(1+\varepsilon_{n}\right)\right)-W_{1}\left(t \gamma^{-1}\right)\right|, \text { for each } n \geq 1 \\
& \stackrel{d}{=} \sup _{0 \leq t \leq 1}\left|\gamma^{-1 / 2}\left(W_{1}\left(t+t \varepsilon_{n}\right)-W_{1}(t)\right)\right|, \text { for } 1<\gamma<\infty \\
& \leq \sup _{0 \leq t \leq 1} \sup _{0<s \leq\left|\varepsilon_{n}\right|} \gamma^{-1 / 2}\left|W_{1}(t+s)-W_{1}(t)\right| \\
& \leq \sup _{0 \leq t \leq 1} \sup _{0<s \leq \varepsilon} \gamma^{-1 / 2}\left|W_{1}(t+s)-W_{1}(t)\right| \text { a.s. } \\
& =O(1)(\varepsilon \log 1 / \varepsilon)^{1 / 2} \text { a.s. } \\
& =o(1) \text { a.s. }
\end{aligned}
$$

by the Lévy modulus of continuity as $\varepsilon \downarrow 0$, and in view of having the last inequality with any $\varepsilon>0$, however small, for all but a finite number of $n$ on account of $\varepsilon_{n} \rightarrow 0$ a.s. as $n \rightarrow \infty$. Thus, via (2.21), we conclude (2.19) under the condition (1.2), and a similar argument yields (2.20) as well under the same condition.

Assuming (1.2) with $\eta=0$, results in having $\varepsilon_{n} \rightarrow 0$ in probability, as $n \rightarrow$ $\infty$. Consequently, and equivalently, every subsequence $\left\{\varepsilon_{n_{k}}\right\}$ contains a further subsequence, say $\left\{\varepsilon_{n_{k(m)}}\right\}$, so that $\varepsilon_{n_{k(m)}} \rightarrow 0$ a.s. as $n_{k(m)} \rightarrow \infty$.

Now to conclude (2.19) in this case, as in the proof above, we arrive at the first inequality with $\varepsilon_{n} \rightarrow 0$ in probability, as $n \rightarrow \infty$, in the expression

$$
\sup _{0 \leq t \leq 1} \sup _{0<s \leq\left|\varepsilon_{n}\right|} \gamma^{-1 / 2}\left|W_{1}(t+s)-W_{1}(t)\right|=: \delta_{n}
$$

and, in order to have (2.19), we wish to conclude that, as $n \rightarrow \infty, \delta_{n} \rightarrow 0$ in probability. This, in turn, is seen to be equivalent to showing that $\delta_{n_{k(m)}} \rightarrow 0$ a.s. 
as $n_{k(m)} \rightarrow \infty$, on account of having $\varepsilon_{n} \rightarrow 0$ in probability as $n \rightarrow \infty$, if and only if, as above, $\varepsilon_{n_{k(m)}} \rightarrow 0$ a.s. as $n_{k(m)} \rightarrow \infty$.

Accordingly, we consider

$$
\begin{aligned}
\delta_{n_{k(m)}} & :=\sup _{0 \leq t \leq 1} \sup _{0<s<\left|\varepsilon_{n_{k(m)}}\right|} \gamma^{-1 / 2}\left|W_{1}(t+s)-W_{1}(t)\right| \\
& \leq \sup _{0 \leq t \leq 1} \sup _{0<s<\varepsilon} \gamma^{-1 / 2}\left|W_{1}(t+s)-W_{1}(t)\right| \text { a.s. } \\
& =O(1)(\varepsilon \log 1 / \varepsilon)^{1 / 2} \text { a.s. } \\
& =o(1) \text { a.s. }
\end{aligned}
$$

by the Lévy modulus of continuity as $\varepsilon \downarrow 0$, and on account of having the last inequality with any $\varepsilon>0$, however small, for all but a finite number of the sequence $\left\{n_{k(m)}\right\}$ that increases to infinity and $\varepsilon_{n_{k(m)}} \rightarrow 0$. This amounts to having $\delta_{n}$ of (2.22) converging to 0 in probability, as $n \rightarrow \infty$, that in turn yields (2.19) under the condition (1.2) with $\eta=0$. A similar argument results in having (2.20) as well under the same condition. This also completes the proof of Lemma 1.

Proof of Theorem 1 With $W_{1}$ and $W_{2}$ as in (2.6), and assuming (1.2) with $\eta=0$, we combine (2.17) with (2.19) and (2.18) with (2.20), and thus conclude Theorem 1 with $T=1$, without loss of generality, i.e., similarly for all fixed $T>0$ as well.

Lemma 2 Under condition (1.2), as $n \rightarrow \infty$, we have

$$
\sup _{0 \leq t \leq 1}\left|\frac{W_{1}\left(n t \gamma^{-1}\left(1+\varepsilon_{n}\right)\right)-W_{1}\left(n t \gamma^{-1}\right)}{(2 n \log \log n)^{1 / 2}}\right|=o(1) \text { a.s. }
$$

as well as

$$
\sup _{0 \leq t \leq 1}\left|\frac{W_{2}\left(n t\left(1-\gamma^{-1}\right)\left(1+\tilde{\varepsilon}_{n}\right)\right)-W_{2}\left(n t\left(1-\gamma^{-1}\right)\right)}{(2 n \log \log n)^{1 / 2}}\right|=o(1) \text { a.s. }
$$

where $\varepsilon_{n}$ and $\tilde{\varepsilon}_{n}$ both converge almost surely to 0 , as indicated in (2.16).

Just like in the case of Lemma 1 , here too, $W_{1}$ and $W_{2}$ can be any generic Wiener processes. The specific forms of (2.24) and (2.25) are only for the sake of convenient reference when proving Theorem 2 and, later on, when indicating the proof of Theorem 3 .

In the proof of Lemma 2, we make use of the following large increment result of Csörgő and Révész $(1979,1981)$ for a standard Wiener process $\{W(t), t \geq 0\}$ : Let $a_{T}(T \geq 0)$ be a monotonically nondecreasing function of $T$ so that $T / a_{T}$ is nondecreasing and $0<a_{T} \leq T$. Define $\beta_{T}=\left\{2 a_{T}\left(\log \frac{T}{a_{T}}+\log \log T\right)\right\}^{-1 / 2}$. Then

$$
\limsup _{T \rightarrow \infty} \sup _{0 \leq t \leq T-a_{T}} \sup _{0 \leq s \leq a_{T}} \beta_{T}|W(t+s)-W(t)|=1 \text { a.s. }
$$


Proof of Lemma 2 We have

$$
\begin{aligned}
& \sup _{0 \leq n t \gamma^{-1} \leq n \gamma^{-1}}\left|W_{1}\left(n t \gamma^{-1}\left(1+\varepsilon_{n}\right)\right)-W_{1}\left(n t \gamma^{-1}\right)\right| \\
& \leq \sup _{0 \leq n t \leq n} \sup _{0 \leq n t\left|\varepsilon_{n}\right| \leq n\left|\varepsilon_{n}\right|}\left|W_{1}\left(n t \gamma^{-1}+n t \varepsilon_{n} \gamma^{-1}\right)-W_{1}\left(n t \gamma^{-1}\right)\right| \\
& \leq \sup _{0 \leq n t \leq n} \sup _{0 \leq s \leq n \varepsilon}\left|W_{1}\left(n t \gamma^{-1}+s \gamma^{-1}\right)-W_{1}\left(n t \gamma^{-1}\right)\right|
\end{aligned}
$$

with any $\varepsilon>0$, however small, for all but a finite number of $n$, on account of $\varepsilon_{n} \rightarrow 0$ a.s. as $n \rightarrow \infty$.

Moreover, with any $0<\varepsilon<1$,

$$
\begin{aligned}
& \sup _{0 \leq n t \leq n} \sup _{0 \leq s \leq n \varepsilon}\left|W_{1}\left(n t \gamma^{-1}+s \gamma^{-1}\right)-W_{1}\left(n t \gamma^{-1}\right)\right| \\
\leq & \sup _{0 \leq n t \leq n-n \varepsilon} \sup _{0 \leq s \leq n \varepsilon}\left|W_{1}\left(n t \gamma^{-1}+s \gamma^{-1}\right)-W_{1}\left(n t \gamma^{-1}\right)\right| \\
& +\sup _{n-n \varepsilon \leq n t \leq n} \sup _{0 \leq s \leq n \varepsilon}\left|W_{1}\left(n t \gamma^{-1}+s \gamma^{-1}\right)-W_{1}\left(n t \gamma^{-1}\right)\right| \\
= & O\left(\left(2 n \varepsilon\left(\log \frac{n}{n \varepsilon}+\log \log n\right)\right)^{1 / 2}\right) \\
= & O\left(\left(2 n \varepsilon \log \frac{1}{\varepsilon}+\varepsilon 2 n \log \log n\right)^{1 / 2}\right) \\
= & \left(\varepsilon \log \frac{1}{\varepsilon}+\varepsilon\right)^{1 / 2} O\left((2 n \log \log n)^{1 / 2}\right) \text { a.s. }
\end{aligned}
$$

as $n \rightarrow \infty$, on applying (2.26) twice.

On combining now (2.28) with (2.27) via letting $n \rightarrow \infty$ and $\varepsilon \downarrow 0$, we arrive at

$$
\sup _{0 \leq t \leq 1} \frac{\left|W_{1}\left(n t \gamma^{-1}\left(1+\varepsilon_{n}\right)\right)-W_{1}\left(n t \gamma^{-1}\right)\right|}{(2 n \log \log n)^{1 / 2}}=o(1) \text { a.s., }
$$

i.e., we have (2.24). Mutatis mutandis, the conclusion of (2.25) is seen to be true as well along similar lines.

Proof of Theorem 2 By (2.14), as $n \rightarrow \infty$,

$$
\sup _{0 \leq t \leq 1}\left|\frac{X_{[n t]}-W_{1}\left(n t \gamma^{-1}\left(1+\varepsilon_{n}\right)\right)}{(2 n \log \log n)^{1 / 2}}\right|=O\left(\frac{\log n}{(2 n \log \log n)^{1 / 2}}\right) \text { a.s. }
$$

Putting (2.24) and (2.29) together, as $n \rightarrow \infty$, we conclude

$$
\sup _{0 \leq t \leq 1}\left|\frac{X_{[n t]}-W_{1}\left(n t \gamma^{-1}\right)}{(2 n \log \log n)^{1 / 2}}\right|=o(1) \text { a.s. }
$$

In a similar fashion, as $n \rightarrow \infty$, one concludes

$$
\sup _{0 \leq t \leq 1}\left|\frac{Y_{[n t]}-W_{2}\left(n t\left(1-\gamma^{-1}\right)\right)}{(2 n \log \log n)^{1 / 2}}\right|=o(1) \text { a.s. }
$$

as well. Consequently, via (2.30) and (2.31), we arrive at having Theorem 2. 


\subsection{Preliminaries and proofs of Theorem $\mathrm{C}$ and Theorem 3}

Given $C=\{C(x)\}_{x \in \mathbb{Z}}$ as in (1.16) with probability law $\mu$ on $\{0,1\}^{\mathbb{Z}}$ as in (1.17), i.e., assuming the $(*)$ condition of (2) of den Hollander (1994), both components of the random walk $\{\mathbf{Z}(n)\}_{n \geq 0}=\{X(n), Y(n)\}_{n \geq 0}$ as in (1.18) with transition probabilities as in (1.19) behave as simple random walks on $\mathbb{Z}$, except for random time delays in the respective total number of horizontal and vertical steps $n_{x}(n)$ and $n_{y}(n)$ in the first $n$ steps of $\mathbf{Z}(n)$ as they are defined in (1.20). In this regard we quote from the first paragraph of Section 2 of den Hollander (ibid.):

\footnotetext{
"Indeed, first $X(n)$ makes a succession of steps until it hits a connected column. Next the walk spends some time on this column, during which $X(n)$ remains fixed and $Y(n)$ makes a succession of steps, until the walk decides to move off the column. Then $X(n)$ again takes over, until it hits a next connected column, etc."
}

Thereby, along the lines of the proof of Ansatz 1 (ibid.), the random walk $\{\mathbf{Z}(n)\}_{n \geq 0}$ as in (1.18) with transition probabilities as in (1.19) can be studied in terms of

$$
\begin{aligned}
\{\mathbf{Z}(n)\}_{n \geq 0} & =\{X(n), Y(n)\}_{n \geq 0} \\
& =\left\{S_{x}\left(n_{x}(n)\right), S_{y}\left(n_{y}(n)\right)\right\}_{n \geq 0},
\end{aligned}
$$

where $S_{x}$ and $S_{y}$ are independent simple random walks, and $n_{x}(n)$ and $n_{y}(n)$ respectively stand for the total number of horizontal and vertical steps in the first $n$ steps of $\mathbf{Z}(n)$ as defined in (1.20).

Given $C=\{C(x)\}_{x \in \mathbb{Z}}$ as in (1.16) with probability law $\mu$ on $\{0,1\}^{\mathbb{Z}}$ as in (1.17), i.e. assuming the $(*)$ condition of (2) of den Hollander (1994), we have (1.21), proved (ibid.) as Ansatz 1 of Shuler (1979), with the conclusions of (1.22). Hence, we may put

$$
n_{x}(n)=n(1 /(1+q))\left(1+\varepsilon_{n}\right)
$$

and, consequently,

$$
n_{y}(n)=n(q /(1+q))\left(1+\varepsilon_{n}\right)
$$

with $\varepsilon_{n} \rightarrow 0 P$-a.s., as $n \rightarrow \infty$.

As a consequence of having (2.32) in combination with (2.33) and (2.34), one can construct two independent standard Wiener processes $\left\{W_{1}(t), t \geq 0\right\}$ and $\left\{W_{2}(t), t \geq 0\right\}$ so that via $[\mathrm{KMT}](1975)$, we arrive at

$$
\sup _{0 \leq t \leq T}\left\|\frac{X([n t])-W_{1}\left(n t(1 /(1+q))\left(1+\varepsilon_{n}\right)\right)}{n^{1 / 2}}, \frac{Y([n t])-W_{2}\left(n t(q /(1+q))\left(1+\varepsilon_{n}\right)\right)}{n^{1 / 2}}\right\|
$$




$$
=O\left(\log n / n^{1 / 2}\right) P-\text { a.s. }
$$

for all fixed $T>0$, as $n \rightarrow \infty$.

Also, under the same conditions that yield (2.33) and (2.34), mutatis mutandis accordingly, the respective statements of Lemmas 1 and 2 of Section 2.1 continue to hold true in the present context.

Thus, on combining the latter version of Lemma 1 with the conclusion of (2.35) with $T=1$, we obtain Theorem $\mathrm{C}$ with $T=1$, without loss of generality, i.e., similarly for all fixed $T>0$ as well (cf. also Remark 5).

Furthermore, the conclusion of Lemma 2 in the present context in combination with that of (2.35) with $T=1$, yields Theorem 3 .

Acknowledgements We wish to thank a number of referees for carefully reading our manuscript, and for their constructive remarks and suggestions that have very much helped us in preparing this revised version of our paper. The research of E. Csáki and P. Révész was supported by Hungarian National Research, Development and Innovation Office - NKFIH K 108615. The research of M. Csörgő was supported by an NSERC Canada Discovery Grant at Carleton University. The research of A. Földes was supported by PSC CUNY Grant, No. 69040-0047.

\section{References}

[1] Bertacchi, D. (2006). Asymptotic behaviour of the simple random walk on the 2-dimensional comb. Electron. J. Probab. 11 1184-1203.

[2] Bertacchi, D. And ZuCCA, F. (2003). Uniform asymptotic estimates of transition probabilities on combs. J. Aust. Math. Soc. 75 325-353.

[3] Csáki, E., Csörgő, M., Földes, A. And RÉvész, P. (2009). Strong limit theorems for a simple random walk on the 2-dimensional comb. Electr. J. Probab. $142371-2390$.

[4] Csáki, E., Csörgő, M., Földes, A. And RÉvész, P. (2011). On the local time of random walk on the 2-dimensional comb. Stochastic Process. Appl. 121 $1290-1314$.

[5] Csáki, E., Csörgő, M., Földes, A. And Révész, P. (2013). Strong limit theorems for anisotropic random walks on $\mathbb{Z}^{2}$. Periodica Math. Hungar. 67 71-94.

[6] Csáki, E., Földes, A. And Révész, P. (2015). Some results and problems for anisotropic random walks on the plane. D. Dawson et al. (eds), Asymptotic 
Laws and Methods in Stochastics, Fields Institute Communications 76 55-75. Springer Science + Business Media New York.

[7] Csörgö, M. And Martsynyuk, Y.V. (2011). Functional central limit theorems for self-normalized least squares processes in regression with possibly infinite variance data. Stochastic Process. Appl. 121 2925-2953.

[8] Csörgő, M. And RÉvÉsz, P. (1979). How big are the increments of a Wiener process? Ann. Probab. 7 731-737.

[9] Csörgő, M. And RÉvész, P. (1981). Strong Approximation in Probability and Statistics. Academic, New York.

[10] Den Hollander, F. (1994). On three conjectures by K. Shuler. J. Statist. Physics 75 891-918.

[11] Dvoretzky, A. And Erdős, P. (1951). Some problems on random walk in space. Proc. Second Berkeley Symposium, pp. 353-367.

[12] ERdős, P. And TAYlor, S.J. (1960). Some problems concerning the structure of random walk paths. Acta Math. Acad. Sci. Hungar. 11 137-162.

[13] Hall, P. And Heyde, C.C. (1980). Martingale Limit Theory and its Application. Academic, New York.

[14] Heyde, C.C. (1982). On the asymptotic behaviour of random walks on an anisotropic lattice. J. Statist. Physics 27 721-730.

[15] Heyde, C.C. (1993). Asymptotics for two-dimensional anisotropic random walks. In: Stochastic Processes. Springer, New York, pp. 125-130.

[16] Komlós, J., Major, P. And Tusnády, G. (1975). An approximation of partial sums of independent rv's and the sample df. I. Z. Wahrsch. verw. Gebiete 32 111-131.

[17] RÉvÉsz, P. (2013). Random Walk in Random and Non-Random Environments, 3rd ed. World Scientific, Singapore.

[18] Shuler, K.E. (1979). Random walks on sparsely periodic and random lattices I. Physica A $9512-34$.

[19] Strassen, V. (1964). An invariance principle for the law of the iterated logarithm. Z. Wahrsch. verw. Gebiete 3 211-226. 
[20] Strassen, V. (1967). Almost sure behaviour of sums of independent random variables and martingales. Proceedings of the Fifth Berkeley Symposium of Mathematical Statistics and Probability, 2, 315-343. University of California Press, Berkeley.

[21] Weiss, G.H. and Havlin, S. (1986). Some properties of a random walk on a comb structure. Physica A 134 474-482. 